\title{
Fatal bleeding in a nasopharyngeal carcinoma patient after concurrent chemoradiation plus cetuximab: a case report
}

This article was published in the following Dove Press journal:

OncoTargets and Therapy

12 June 2013

Number of times this article has been viewed

\section{LingYan Zheng' \\ SenXiang Yan' \\ DanFang Yan' \\ JingSong Yang' \\ YiXiang Wang ${ }^{2}$}

'Department of Radiation Oncology, First Affiliated Hospital, College of Medicine, Zhejiang University, Hangzhou, People's Republic of China; ${ }^{2}$ Department of Diagnostic Radiology and Organ Imaging, Prince of Wales Hospital, Hong Kong
Correspondence: SenXiang Yan Department of Radiation Oncology, First Affiliated Hospital, College of Medicine, Zhejiang University, 866 Yuhangtang Road, Hangzhou 310058, People's Republic of China Email yansenxiang@zju.edu.cn
Abstract: Carotid blowout syndrome (CBS) refers to the clinical signs and symptoms related to rupture of the carotid artery (CA) and its branches, which mainly results from malignant invasion of the CA by head and neck cancers. Here, we present a 46-year-old male patient who suffered from nasopharyngeal carcinoma and was treated with a combination of chemoradiation and cetuximab. The patient was stage IVb (T4N2M0) clinically, with encasement of the left internal carotid artery, as shown on pretreatment magnetic resonance imaging. Three months after completion of radiotherapy, the patient died of sudden massive epistaxis. CBS is a lethal complication of nasopharyngeal carcinoma, so the risk of CBS should be carefully assessed in patients with imaging showing $\mathrm{CA}$ encasement. Till now, the precise prediction and prevention of CBS remain to be explored.

Keywords: nasopharyngeal carcinoma, carotid blowout syndrome, diagnosis, cetuximab, prevention

\section{Introduction}

In many parts of Asia, including Southern China and Southeast Asia, nasopharyngeal carcinoma (NPC) is the most common head and neck cancer. Recent advances in treatment, including intensity-modulated radiation therapy (IMRT) and combined chemoradiation, have contributed to considerable improvement in local control of this disease. However, there are more and more cases with a history of carotid blowout syndrome (CBS) reported in NPC patients. From our single institution's statistics, the overall 3-year locoregional control rate for 200 patients with NPC has reached as high as $98 \%$, yet at the same time three cases (1.5\%) developed fatal bleeding due to CBS. In other words, $2 \%$ locoregional failure at 3 years corresponded to $1.5 \%$ fatal bleeding from our series of patients with NPC. Thus, early prediction and prevention of the occurrence of CBS-related bleeding would translate into clinical benefits in those patients who might otherwise be cured. According to the literature, the mortality and neurovascular morbidity rates associated with CBS can be as high as $40 \%-60 \%{ }^{1}$ Both tumor invasion and radiation-related complications are considered potential causes of CBS, and its prediction and prevention remain a problem in clinical practice.

Herein, we report a case of NPC with fatal CBS 3 months after combined chemoradiation and cetuximab treatment. Informed consent to the treatment was obtained from the patient, and the Institutional Review Board of the authors' hospital approved the retrospective report of this case. 


\section{Case report}

A 46-year-old male was diagnosed with NPC in 2011, and staged as IVb (T4N2M0) according to the 7th edition of the American Joint Committee on Cancer after staging workup including magnetic resonance imaging (MRI), computed tomography (CT), and bone scan. Pretreatment MRI of the nasopharynx (Figure 1A-D) revealed a primary lesion predominantly occupying the left nasopharynx and extending into the left masticator space, with encasement of the left internal carotid artery. The patient received curative treatment, which consisted of IMRT and concurrent chemotherapy with cisplatin plus cetuximab. An initial loading dose of cetuximab at $600 \mathrm{mg} / \mathrm{m}^{2}$ was given intravenously 1 day before IMRT, then cetuximab was given weekly at a dose of $400 \mathrm{mg} / \mathrm{m}^{2}$ for six continuous cycles. Starting from the first day of IMRT, cisplatin at a dose of $100 \mathrm{mg} / \mathrm{m}^{2}$ was given, and was repeated at a 3-week interval. With IMRT, 6976 cGy over 32 daily fractions was given to the gross tumor volumes, including the primary lesion and enlarged lymph nodes. The high-risk clinical target volume, including areas adjacent to the primary lesion and lymphadenopathy, received 6104 cGy over 28 fractions, and the low-risk clinical target volume of the lower neck received 5264 cGy over 28 fractions. Following the initial concurrent chemoradiotherapy, the patient was given three cycles of adjuvant chemotherapy with cisplatin at
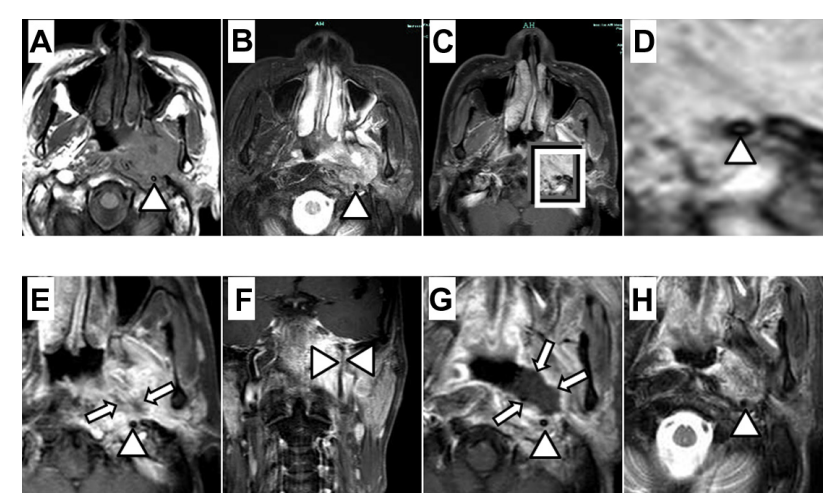

Figure I (A-D) MRI study of the left-sided nasopharyngeal carcinoma before combined CRT plus cetuximab therapy, showing extensive involvement of the left prevertebral and the pterygoid muscles with encasement of the left ICA (A: axial TIWI, B: T2WI with fat suppression, C: Gd ${ }^{+}$TIWI; D: magnification of the lesion in $\mathbf{C})$. (E and $\mathbf{F}$ ) One month after treatment (E: Gd ${ }^{+} \mathrm{TIWI}$ shows patchy unenhanced areas in the center of the lesion, representing foci of necrosis; $\mathbf{F}$ : coronal Gd ${ }^{+}$TIWI shows the ICA meandering through the lesion). ( $\mathbf{G}$ and $\mathbf{H}$ ) Three months after treatment (G: Gd ${ }^{+}$TIWI shows large unenhanced area, bordering the ICA, in the lesion; $\mathbf{H}$ : T2WI shows the unenhanced area to be of moderately high signal intensity, representing coagulative necrosis of the lesion).

Notes: Arrowheads indicate the left ICA; arrows indicate coagulative necrosis in the lesion.

Abbreviations: MRI, magnetic resonance imaging; ICA, internal carotid artery; CRT, chemoradiation; TIWI, $\mathrm{T}_{1}$-weighted imaging; T2WI, $\mathrm{T}_{2}$-weighted imaging; $\mathrm{Gd}^{+}$TIWI, gadolinium-enhanced $\mathrm{T}_{1}$-weighted imaging.
$80 \mathrm{mg} / \mathrm{m}^{2}$ on day 1 and 5 -fluorouracil at $1000 \mathrm{mg} / \mathrm{m}^{2}$ on days $1-5$ every 4 weeks.

The patient tolerated the treatment, with grade II hematological and mucosal reactions that did not lead to interruption of radiotherapy. Three weeks after initiation of cetuximab, an acneiform rash developed and aggravated to grade III another 2 weeks later. MRI scans taken 1 and 3 months after completion of radiotherapy are shown in Figure 1E-H, revealing gradual tumor shrinkage with rapidly spreading coagulative necrosis adjacent to the left internal carotid artery. On September 1, 2011 and 20 days after the last MRI scan (Figure $1 \mathrm{G}$ and $\mathrm{H}$ ), the patient experienced excretion of a large quantity of yellow-grayish debris from the mouth and "felt much better than before," as described by his relatives. Then on the next day, the patient had a sudden massive epistaxis. After immediate resuscitation and a large amount of compressive oronasopharyngeal packing, the vital signs were not stabilized, and he died before there was enough time to carry out effective procedures to achieve hemostasis.

\section{Discussion}

Predisposing factors for CBS in a patient with NPC include advanced tumor stage and radiation-induced complications such as premature atherosclerosis, adventitial fibrosis, and wall necrosis. In cases of CBS occurring early after radiotherapy, the pre-existing tumor invasion of the carotid artery (CA) and defects in the arterial wall following tumor shrinkage might play major roles. Pretreatment MRI scans (Figure 1A-D) in the current case showed tumor encasement of the $\mathrm{CA}$, which suggested the possibility of an invasion into the arterial wall. Clinically, the precise and noninvasive diagnosis of arterial wall invasion or defects remains problematic. CT, MRI, and ultrasound (US) have been used to evaluate the integrity of the CA wall. ${ }^{2} \mathrm{CT}$ and MRI criteria for detecting CA involvement include loss of fat or fascial planes between the malignant neoplasm and CA, and the circumference of tumor attachment around the CA..$^{2-6}$ Gritzmann et al found that tumor encasement of carotid vessels was an indirect predictor of the likelihood of invasion into the CA. Encasement of more than $180^{\circ}$ circumference highly suggested the existence of invasion. ${ }^{7}$ Yousem et al reported that malignant lesion with $270^{\circ}$ or less circumferential involvement was suggestive of lack of invasion. They used this rule to predict CA invasion with MRI images: circumferential involvement of $270^{\circ}$ or less of the arterial wall as no invasion, and more than $270^{\circ}$ as invasion. According to the above criteria, the sensitivity, specificity, accuracy, negative predictive value, and positive predictive value of MRI were $100 \%, 88 \%, 90 \%, 100 \%$, and 
$70 \%$, respectively, compared with pathology. ${ }^{5}$ They made the conclusion that the efficacy of MRI or CT largely depends on the aforementioned criteria for evaluation of CA invasion by a tumor. But such criteria are inherently imprecise because of their indirectness. On the contrary, US can directly show the absence of echogenicity of the vascular wall or the loss of its bright hyperechogenic layer, which was taken as a sign of tumoral infiltration. ${ }^{8}$ Thus, many authors considered US an effective and reliable method for investigating the potential invasion of CA. Nonetheless, another study has demonstrated that cancer seldom invades the lumen of the CA and usually invades the adventitia or external elastic membrane. ${ }^{9}$ As a result, the value of noninvasive diagnosis of arterial invasion by the current imaging modalities is still limited.

The optimal management for patients with NPC possibly invading the CA is controversial. Radiotherapy is reported to lead to adventitial fibrosis, permanent atherosclerosis, subendothelial vacuolization, and edema, which weaken the arterial wall. Radiotherapy has been associated with a sevenfold increase in the risk of CA rupture in patients with head and neck cancers. ${ }^{10}$ Cengiz et al reported that eight (17.3\%) patients had CBS, and seven (15.2\%) patients died of bleeding from the $\mathrm{CA}$ in their study for locally recurrent head and neck tumors treated with reirradiation using stereotactic body radiotherapy. They discovered that this fatal syndrome occurred only in patients with tumor surrounding half or more of the CA wall and the CA receiving $100 \%$ of the prescribed dose. ${ }^{11}$ As for our case, the left CA around the site of encasement received a mean dose of $102 \%$, and a maximal dose of $104 \%$ of the prescribed 6976 cGy (Figure 2). We suggest that more caution should be given dosimetrically to the CA during treatment planning to prevent CBS. Our patient was also given combined chemoradiotherapy and cetuximab, a monoclonal antibody-targeting epidermal growth-factor receptor. Although clinical data have shown that cetuximab can be safely combined with concurrent cisplatin and IMRT in the curative treatment of locoregionally advanced NPC, the occurrence of massive hemorrhage has not been observed to date. ${ }^{12}$ The combined treatment in the current case might have caused rapid shrinkage of the primary lesion and the formation of a large volume of coagulative necrosis, which induced the development of CBS. In a phase II study of sunitinib, ${ }^{13}$ a multi-tyrosine kinase inhibitor, in patients with NPC who had previously received high-dose radiation, hemorrhagic events occurred in nine out of 13 patients (64\%), including epistaxis in six, hemoptysis in three, and hematemesis in two patients. Two patients with local tumor invasion into the carotid sheath developed fatal epistaxis/hematemesis within the first cycle of

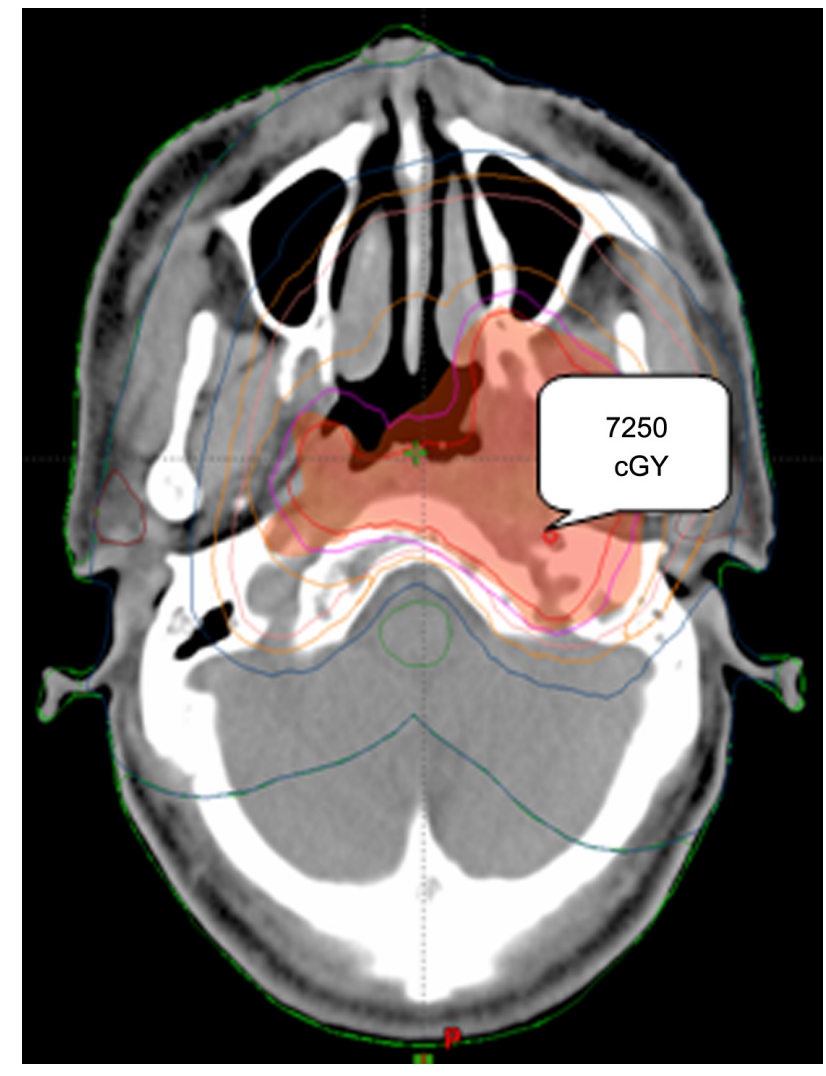

Figure 2 A dosimetric map around the encased carotid artery.

Note: A point dose of 7250 cGy (104\% of the prescribed dose) is indicated within the artery.

sunitinib. In one of the two patients, significant tumor shrinkage $(>60 \%)$ was noted on the MRI study carried out before the fatal hemorrhagic event. The tumor necrosis resulted in the naked internal CA exposing to the nasopharyngeal cavity. In our case, the series of MRI studies disclosed encasement of the artery before (Figure 1A-D), and rapidly spreading necrosis after, the treatment (Figure $1 \mathrm{G}$ and $\mathrm{H}$ ). Therefore, we suggest that pre-existing invasion of the arterial wall before, and direct exposure of the injured artery to the nasopharyngeal cavity after, the treatment might have contributed to the subsequent CBS with fatal epistaxis. Furthermore, another mechanism called "radiation recall" has been reported after targeted therapies (cetuximab, bevacizumab, mammalian target of rapamycin inhibitors). ${ }^{14-16}$ Some targeted agents possibly increase the risk of radiation-related vascular inflammation and injury. Therefore, we propose the avoidance of the addition of cetuximab to chemoradiotherapy in patients with tumor possibly invading the CA.

What can be done in the case of an impending CBS? Current treatment options involve either surgical or endovascular procedures. Ligation, resection, and/or reconstruction of the $\mathrm{CA}$ are the common surgical methods. 
But these surgical procedures result in major neurologic complications postoperatively, especially in the setting of ligation. Also, surgical intervention in such a deep and complex site is rather challenging and risk-taking. Alternatively, researchers have turned to interventional endovascular measures for more efficacious management. Endovascular approaches such as occlusion and stent grafts of the affected arteries have been applied in emergency treatment in the case of uncontrolled carotid bleeding. Detachable balloons, coils, or microparticles have been utilized to occlude the bleeding arteries. However in $15 \%-20 \%$ of patients, these procedures can induce delayed ischemic cerebral complications, resulting from an incomplete circle of Willis and thromboembolism associated with an acutely occluded CA, especially in those patients with common CA or internal CA invasion. ${ }^{1,17}$ Therefore, the use of arterial stents to repair rather than to occlude the artery has been advocated in recent years. A recent report has it that the use of endovascular stents resulted in only one episode of transient ischemia (8\%) and a mortality of $0 \%{ }^{16}$

\section{Conclusion}

CBS is a lethal complication of NPC with arterial invasion. The risk of $\mathrm{CA}$ invasion and subsequent rupture should be well assessed in patients with imaging showing CA encasement. Furthermore, it is imperative to develop a safer and more effective endovascular procedure to prevent the occurrence of CBS.

\section{Disclosure}

The authors report no conflicts of interest in this work.

\section{References}

1. Chaloupka JC, Putman CM, Citardi MJ, Ross DA, Sasaki CT. Endovascular therapy for the carotid blowout syndrome in head and neck surgical patients: diagnostic and managerial considerations. AJNR AM J Neuroradiol. 1996;17:843-852.

2. Langman AW, Kaplan MJ, Dillon WP, Gooding GA. Radiologic assessment of tumor and the carotid artery: correlation of magnetic resonance imaging, ultrasound, and computed tomography with surgical findings. Head Neck. 1989;11:443-449.
3. Rothstein SG, Persky MS, Horh S. Evaluation of malignant invasion of the carotid artery by CT scan and ultrasound. Laryngoscope. 1988;98: 321-324.

4. Heppt W, Lenarz T, Gademann G, Fritz P, Gückel F, Born IA. Nachweis von Muskel-, Venen- und Arterieninfiltrationen zervikaler Lymphknotenmetastasen [Detection of muscle, vein and artery infiltration of cervical lymphatic metastases.] Laryngorhinootologie. 1989;68:419-424. German.

5. Yousem DM, Hiroto H, Hurst RW, et al. Carotid artery invasion by head and neck masses: prediction with MR imaging. Radiology. 1995;195: 715-720.

6. Yoo GH, Hocwald E, Korkmaz H, et al. Assessment of carotid artery invasion in patients with head and neck cancer. Laryngoscope. 2000;110: 386-390.

7. Gritzmann N, Grasl MC, Helmer M, Steiner E. Invasion of the carotid artery and jugular vein by lymph node metastases: detection with sonography. AJR Am J Roentgenol. 1990;154:411-414.

8. Zaragoza L, Sendra F, Solano J, Garrido V, Martínez-Morillo M. Ultrasonography is more effective than computed tomography in excluding invasion of the carotid wall by cervical lymphadenopathies. Eur J Radiol. 1993;17:191-194.

9. Huvos AG, Leaming RH, Moore OS. Clinicopathologic study of the resected carotid artery. Analysis of sixty-four cases. Am J Surg. 1973;126: 570-574.

10. Maran AG, Amin M, Wilson JA. Radical neck dissection: a 19 year experience. J Laryngol Otol. 1989;103:760-764.

11. Cengiz M, Özyiğit G, Yazici G, et al. Salvage reirradiaton with stereotactic body radiotherapy for locally recurrent head-and-neck tumors. Int J Radiat Oncol Biol Phys. 2011;81:104-109.

12. Ma BB, Kam MK, Leung SF, et al. A phase II study of concurrent cetuximab-cisplatin and intensity-modulated radiotherapy in locoregionally advanced nasopharyngeal carcinoma. Ann Oncol. 2012;23: 1287-1292.

13. Hui EP, Ma BB, King AD, et al. Hemorrhagic complications in a phase II study of sunitinib in patients of nasopharyngeal carcinoma who has previously received high-dose radiation. Ann Oncol. 2011;22: 1280-1287.

14. Law AB, Junor EJ. Chemotherapy-induced recall of cetuximab and radiation skin reaction. Clin Oncol ( $R$ Coll Radiol). 2009;21:77-78.

15. Saif MW, Ramos J, Knisely J. Radiation recall phenomenon secondary to bevacizumab in a patient with pancreatic cancer. JOP. 2008;9: 744-747.

16. Bourgier C, Moldovan C, Soria JC, Deutsch E. Total recall of radiotherapy with mTOR inhibitors: a novel and potentially frequent side effect? Ann Oncol. 2011;22:485-486.

17. Lesley WS, Chaloupka JC, Weigele JB, Mangla S, Dogar MA. Preliminary experience with endovascular reconstruction for the management of carotid blowout syndrome. AJNR Am JNeuroradiol. 2003;24: 975-981.
OncoTargets and Therapy

\section{Publish your work in this journal}

OncoTargets and Therapy is an international, peer-reviewed, open access journal focusing on the pathological basis of all cancers, potential targets for therapy and treatment protocols employed to improve the management of cancer patients. The journal also focuses on the impact of management programs and new therapeutic agents and protocols on

\section{Dovepress}

patient perspectives such as quality of life, adherence and satisfaction. The manuscript management system is completely online and includes a very quick and fair peer-review system, which is all easy to use. Visit http://www.dovepress.com/testimonials.php to read real quotes from published authors. 